\title{
OPEN Geometric Control of Cell Migration
}

SUBJECT AREAS:

MESENCHYMAL

MIGRATION

ASSAY SYSTEMS

EXTRACELLULAR MATRIX

CELLULAR MOTILITY

Received

14 March 2013

Accepted

13 September 2013

Published

3 October 2013

Correspondence and requests for materials should be addressed to C.C.H. (hocc@ucmail. uc.edu)

\author{
Bo Chen' ${ }^{1}$ Girish Kumar ${ }^{12}$, Carlos C. Co' \& Chia-Chi Ho'
}

\author{
${ }^{1}$ Chemical \& Materials Engineering Program, University of Cincinnati, Cincinnati, OH 45221-0012, USA, ${ }^{2}$ Division of Biology, \\ Center for Devices and Radiological Health, US Food \& Drug Administration, Silver Spring, MD 20993, USA.
}

Morphological polarization involving changes in cell shape and redistribution of cellular signaling machinery, initiate the migration of mammalian cells. Golgi complex typically localizes in front of the nucleus, and this frontwards polarization has been proposed to be involved in directional migration. However, the sequence of events remains unresolved. Does Golgi polarization precede directional migration or vice-versa? We address this question by constraining cells to specific areas and shapes then tracking their motile behavior and the spatio-temporal distribution of Golgi apparatus upon release. Results show that while the position of the Golgi complex depends on the cell geometry, the subcellular localization of the Golgi complex does not define the cell's leading edge. Cells constrained within elongated geometries exhibit polarized extension of lamellipodia and upon release, migrate preferentially along the long axis of the cell. Minimally constrained cells released from larger areas however, exhibit retarded migration regardless of lamellipodia protrusion activity. 
migration arise from the polarization of Golgi relative to the nucleus or the up-regulation of lamellipodia extension at the leading edge?

Here we address these fundamental questions using micropatterned polyelectrolyte self-assembly ${ }^{38,39}$ to first regulate the size and shape of individual cells then non-invasively releasing them from confinement to observe their subsequent migratory behavior.

\section{Results}

Cell confined within smaller areas have greater migration tendency upon release. To examine the effects of cell spreading area on motility, we first confine NIH 3T3 fibroblasts onto cell adhesive islands on tissue culture dishes defined by microcontact printing surrounding regions with cell-resistant poly(OEGMA/
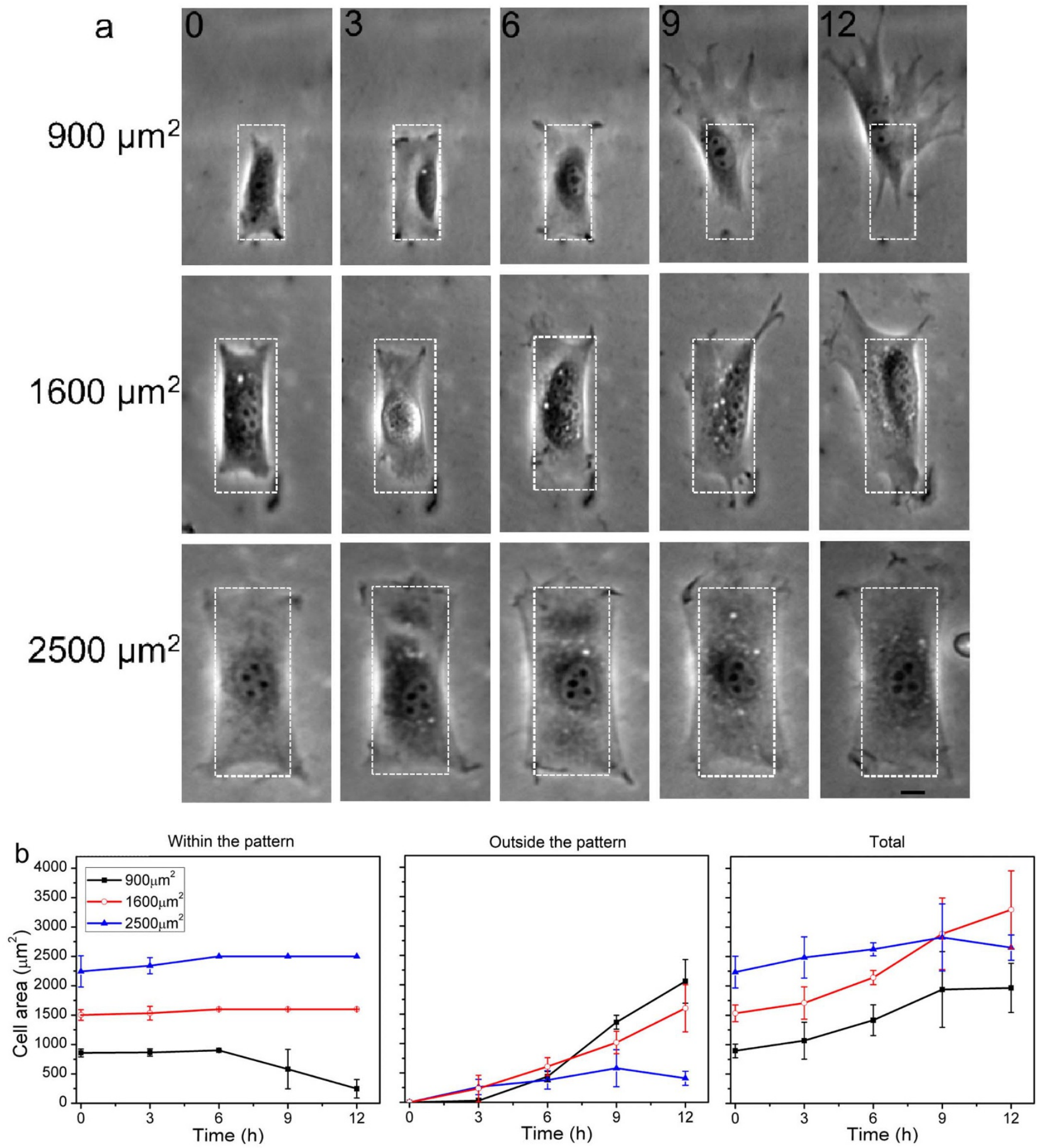

Figure $1 \mid$ Cell migration tendency is dependent on spreading area. (a) Time lapse images (in hours) show the motility of NIH 3T3 fibroblasts upon release from the rectangular shaped adhesive islands with different areas. Scale bar: $10 \mu \mathrm{m}$. Cells on $900 \mu \mathrm{m}^{2}$ or $1600 \mu \mathrm{m}^{2}$ adhesive islands migrate out from either of the short edges. Images are oriented with the short edge of migration directed upwards. (b) Quantification of total cell area, cell area within the pattern and cell area outside of the pattern upon release at time $=0 \mathrm{~h}$ (Mean \pm S.D. calculated from three independent experiments). 
MA). Within 12 hours, 3T3 fibroblasts spread to assume the size and shape of the 900,1600 and $2500 \mu \mathrm{m}^{2}$ rectangular islands, designed to mimic the elongated spindle-shaped morphology of fibroblasts observed in $v i v o^{40}$. After defining the size and shape of the cells, we released them from confinement by adding media-soluble chitosan ${ }^{39}$ which gets adsorbs onto the cell-resistant poly(OEGMA/MA) rendering these surrounding regions cell-adhesive. Cells confined in $900 \mu \mathrm{m}^{2}$ or $1600 \mu \mathrm{m}^{2}$ rectangles spread to fully occupy the available area, whereas cells confined within $2500 \mu \mathrm{m}^{2}$ rectangles spread to areas typically $10 \%$ smaller than the constraining rectangle. The relationship between cell migration tendency and initial spreading area is shown in Figure 1 for NIH 3T3 cells confined to similar rectangles of varying area. Irrespective of their confined area, cells all exhibit active lamellipodia extension at the corners of the rectangles. However, only cells from $900 \mu \mathrm{m}^{2}$ adhesive islands move out of confinement within 12 hours after release. We quantified the effect of initial cell area on cell movement upon release by tracking cell areas within and outside the initially confining rectangles over time. As Figure $1 \mathbf{b}$ shows, cells with initial area of $900 \mu \mathrm{m}^{2}$ retain almost no portion of their body within the initial region of confinement after 12 hours, indicating migration out of the confinement. In contrast, cells with initial confinement areas of 1600 and $2500 \mu \mathrm{m}^{2}$ retain full coverage of their initial region of confinement. The increased cell area outside the pattern is due to overall cell spreading as the increased in total area shows. NIH 3T3 fibroblasts initially confined within $900 \mu \mathrm{m}^{2}$ islands of other geometries also exhibit greater migration tendencies when released compared to cells released from $2500 \mu \mathrm{m}^{2}$ islands of the same geometry (Supplementary Figure S1).

These results show that spreading area alone can influence motility with smaller spreading areas correlated with higher cell motility. In contrast to earlier reports, the cell shape and areas are modulated here without altering the local interaction between the cell and substrate prior to release. Earlier conclusions of reduced average motility on substrates with very strong adhesion resulting from increased integrin expression ${ }^{15}$, binding affinities ${ }^{17}$, ECM concentrations ${ }^{12,19}$, or rigidity ${ }^{41}$ may have confounding effects due to changes in spreading area.

Physical confinement to smaller spreading area suppresses the formation of stress fibers. Cell spreading over larger areas have been correlated with higher overall cell tension ${ }^{42}$. In turn higher tension has been shown to enhance lamellipodia extension ${ }^{43}$ and therefore a greater tendency to migrate. However, focal adhesions also increase in direct proportion to spreading area and is driven by cytoskeletal tension ${ }^{23,44}$. Thus, it was unclear how cell area would affect cell motility.

Figure 2 shows the actin structure and extension of lamellipodium for NIH 3T3 fibroblasts confined to similar rectangular shapes with different areas. Actin structures were most pronounced at the cell periphery along the borders of the confining patterns. As expected, cells confined to smaller adhesive islands $\left(900 \mu \mathrm{m}^{2}\right)$ exhibit
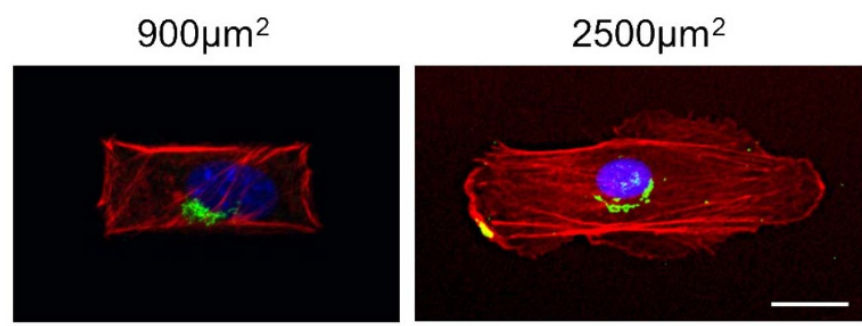

Figure 2 Confinement to smaller spreading area suppresses actin stress fibers. NIH 3T3 cells confined within rectangular shapes with area of $900 \mu^{2}$ (left panel) or $2500 \mu^{2}$ (right panel), respectively. Phalloidin, anti-Golgi, and DAPI were used to label actin filaments (red), Golgi apparatus (green), and nuclei (blue). The scale bar corresponds to $20 \mu \mathrm{m}$. actin cytoskeletons with little to no stress fibers in contrast to their counterparts in larger adhesive islands $\left(2500 \mu \mathrm{m}^{2}\right)$, which exhibit organized actin stress fibers suggestive of higher mechanical tension $^{42,45-47}$. These organized actin filaments are accompanied by more active lamellipodia protrusions from the edges and corners observed for the larger $2500 \mu \mathrm{m}^{2}$ cells. If lamellipodia extension was the only determinant of cell motility, the larger cells would have migrated out of confinement more rapidly. Our observation that these larger cells exhibit negligible migration upon release suggests that the focal adhesions anchoring the cells to the extracellular matrix on the substrate overwhelmingly exceed the traction imparted by the actively extended lamellipodia. These findings extend to cells confined in other 900 and $2500 \mu \mathrm{m}^{2}$ geometries (Supplementary Figure S2).

Geometric confinement influences Golgi apparatus polarization and orientation. The position of the Golgi apparatus relative to the nuclei has been proposed to indicate cell polarity with the Golgi apparatus oriented towards the leading edge $e^{25}$. Cell shape alone has been shown to influence the polarization of mammalian cells on teardrop-shaped adhesive islands, with polarization towards the blunt end ${ }^{48,49}$. However, the independent role of cell spreading area and cell morphology on polarization of Golgi apparatus remains unanswered.

To quantify the effects of cell spreading areas and geometry on Golgi orientation, we analyzed the distribution of Golgi in fibroblast cells confined within 900,1600 and $2500 \mu \mathrm{m}^{2}$ rectangular islands (Figure 3a, b). Cells within the rectangular islands adopt the elongated confining geometry with an average aspect ratio $2.5 \pm 0.3$. We divided the periphery of the nucleus by two intersecting perpendicular diagonal lines centered at the centroid of the nucleus and classified the location of Golgi apparatus as $\mathbf{S}$ when the majority of Golgi orient toward the short edge, $\mathrm{L}$ when the majority of Golgi orient toward on the long edge $\mathrm{L}$, and $\mathbf{O}$ when the majority of the Golgi overlaps with the nucleus. Cells with different spreading areas preferentially orient their Golgi apparatus toward the long edge of the rectangle (Figure 3c). This preference increases with increasing cell spreading area with $82 \%$ of $2500 \mu \mathrm{m}^{2}$ cells orienting their Golgi toward the long edge. Cells with the Golgi apparatus oriented toward the short edge exhibit atypical nuclear alignment with the long axis of the nucleus orienting more perpendicularly to the long axis of the cell (Figure 3b). The splitting of Golgi apparatus to both short edges occurred on rare occasions (observed for 2 of 93 and 3 of 70 cells for $900 \mu \mathrm{m}^{2}$ and $2500 \mu \mathrm{m}^{2}$ rectangles, respectively) and these were not included in the analysis.

To examine the localization of Golgi apparatus, the angle over which Golgi is distributed and quantified for all cells with either short edge (S) or long edge (L) orientation. As Figure 3d shows, the Golgi apparatus cluster over more compact spread angles for cells with smaller spreading areas. These results show that cell size and shape influence the distribution of Golgi. Cell elongation polarizes the Golgi apparatus toward the long edge while cell area impacts the localization of Golgi apparatus. Since the Golgi apparatus is generally located in front of the nucleus towards the leading edge of migratory $\mathrm{cells}^{36}$, the initial direction of cell movement upon release may be expected to be directed horizontally outwards from the long edges.

Directional membrane protrusions mediate initial movement. To investigate whether the increased bias of Golgi towards the long edge versus the short edge lead to preferential migration from the long edge, cells were released by rendering the background region cell adhesive through adsorption of chitosan added to the media ${ }^{39}$. Before release, cells confined within elongated patterns align their actin cytoskeleton diagonally or along the long axis of the cell body (Figure 4a). Although the Golgi of the confined cells are predominantly located close to one of the long edges of $900 \mu \mathrm{m}^{2}$ rectangles ( $48 \%$, Figure $3 c$ ) compared to the short edge (32\%, Figure $3 c)$, we observed that cells extend lamellipodium predominantly from 


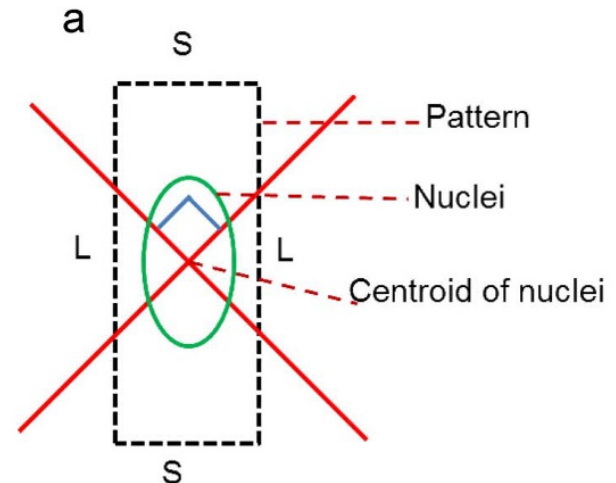

b

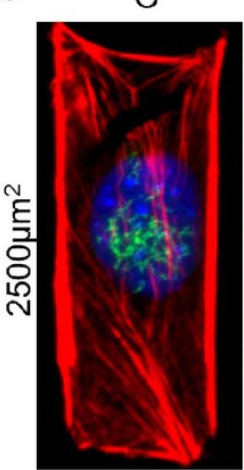

$\mathrm{L}$
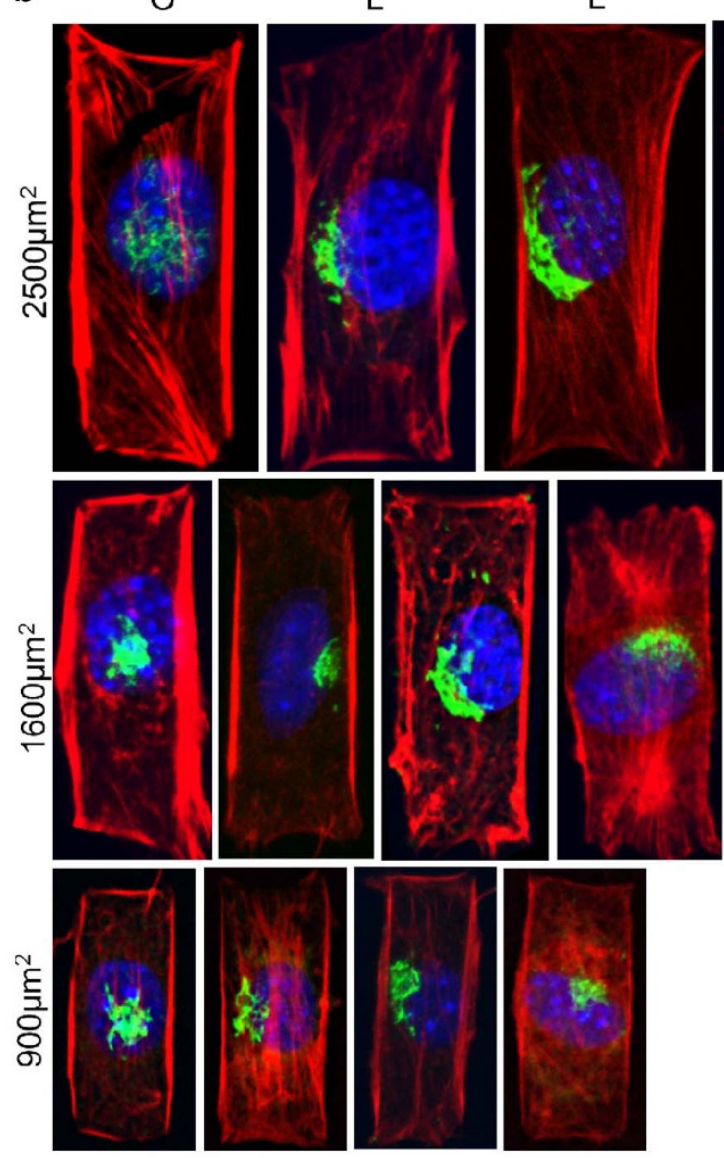
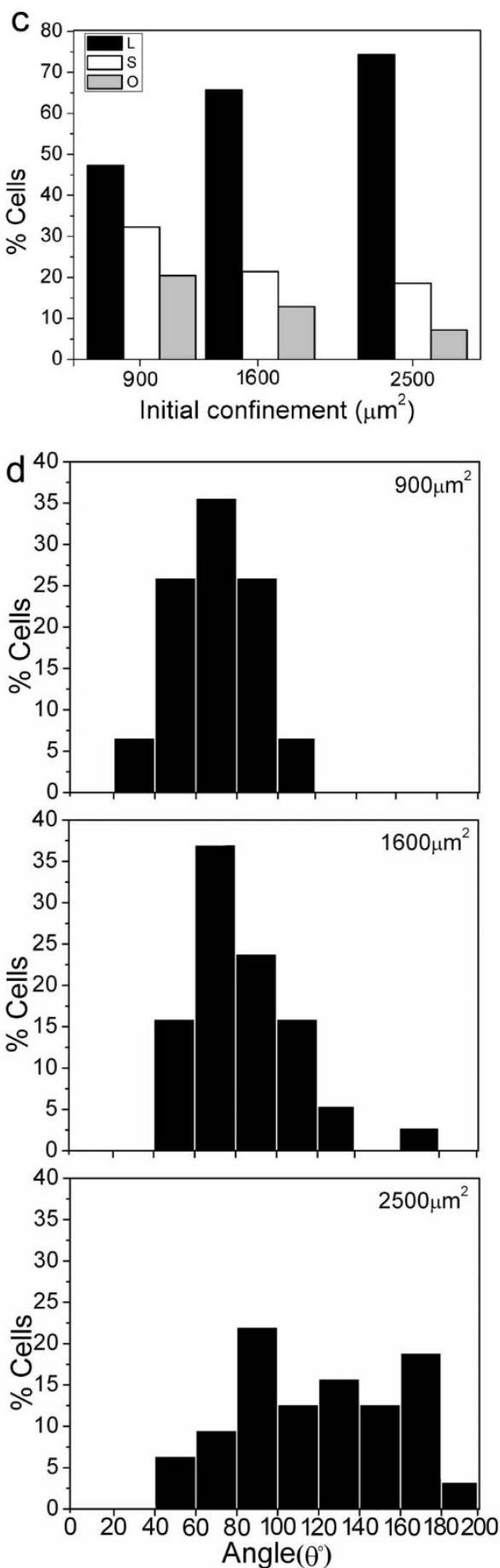

Figure $3 \mid$ Role of initial confinement on polarization and orientation of Golgi apparatus. (a) Schematic diagram of Golgi orientation toward either the long (L) or short edges (S) of the confining pattern. (b) Distribution of Golgi apparatus in NIH 3T3 fibroblasts confined within rectangular shaped islands. Phalloidin, anti-Golgi, and DAPI were used to label actin filaments (red), Golgi apparatus (green), and nuclei (blue). (c) Statistical summaries of the Golgi orientation for fibroblasts on rectangular patterns $\left(\mathrm{N}=93,70,70\right.$ cells for 900,1600 and $2500 \mu \mathrm{m}^{2}$, respectively) On the nucleus $(\mathrm{O})$, short (S) and long (L) edges represent the position of Golgi apparatus relative to the nuclei. Excluding $\mathrm{O}$ cases, cells exhibit preferential organization of Golgi apparatus along the long edges of $900 \mu \mathrm{m}^{2}(\mathrm{p}=0.065), 1600 \mu \mathrm{m}^{2}(\mathrm{p}=0.00004)$, and $2500 \mu \mathrm{m}^{2}(\mathrm{p}=0.000002)$ adhesive islands. (d) Angular distributions of Golgi apparatus for fibroblast cells on rectangular patterns with different areas. The angular distribution of Golgi apparatus were $86.5^{\circ} \pm$ $30^{\circ}, 91.4^{\circ} \pm 34^{\circ}$, and $126.5^{\circ} \pm 41^{\circ}$ (mean \pm SD), for cells on $900 \mu \mathrm{m}^{2}(\mathrm{n}=73), 1600 \mu \mathrm{m}^{2}(\mathrm{n}=67)$, and $2500 \mu \mathrm{m}^{2}(\mathrm{n}=65)$ adhesive islands, respectively. Heteroscedastic $\mathrm{t}$-test of sample means for each pair of island sizes yield the following $\mathrm{p}$ values $900 / 1600 \mathrm{p}=0.37,900 / 2500 \mathrm{p}=2.4 \times 10^{-9}, 1600 / 2500 \mathrm{p}$ $=3.8 \times 10^{-7}$.

the corners 4 hours after release (Figure 4a). Tracking the centroid of individual fibroblasts 4,8 , and 12 hours after release (Figure 4 b) reveals that this biased extension of lamellipodia leads to predominantly vertical translocation wherein cells move out principally from one of the short edges of the rectangles. These findings show that the initial elongated cell shape influences the location of the initial membrane protrusions and biases the direction of initial cell movement along the long axis. 
a
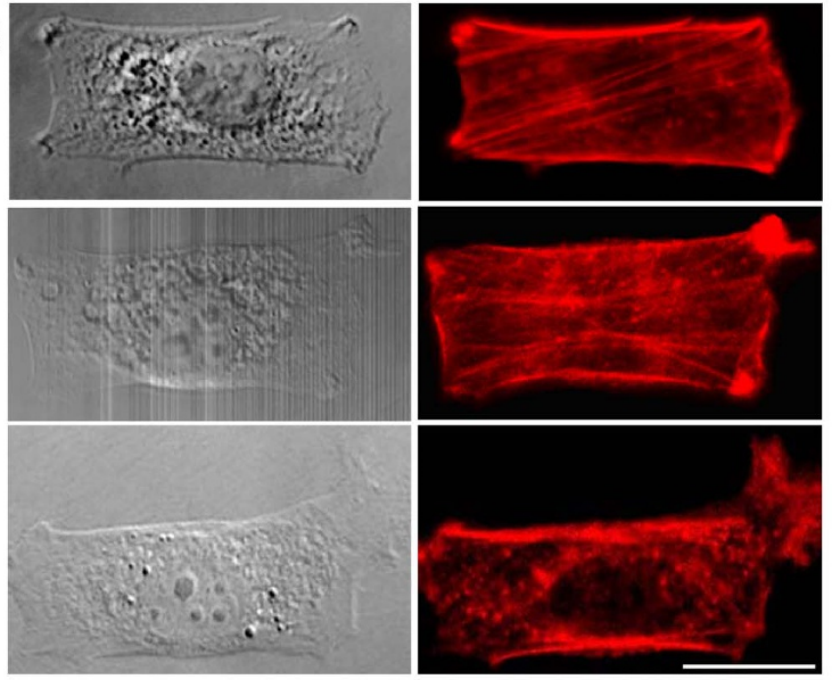

b

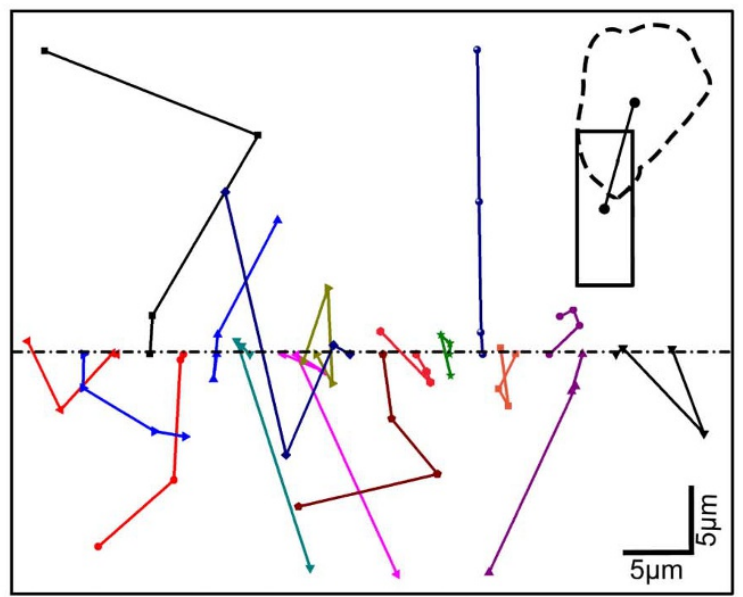

Figure $4 \mid$ Preferential lamellipodial extensions in the corners determine the direction of initial cell movement. (a) NIH $3 \mathrm{~T} 3$ fibroblast cells released from rectangular shaped adhesive islands with area of $900 \mu \mathrm{m}^{2}$ preferentially extend their lamellipodia from the corner. Distribution of actin filament (red) and the corresponding phase contrast images at release $\mathrm{t}=0$ (top row) and 4 hours after release. The scale bar corresponds to $20 \mu \mathrm{m}$. (b) Quantification of the movement of NIH 3T3 cells after release from $900 \mu \mathrm{m}^{2}$ rectangular islands. The starting position is shifted $3 \mu \mathrm{m}$ horizontally to show each trace clearly and each trace (and color) indicates a separate cell. The symbols correspond to the position of the centroids of cells at 4 hours intervals and the lines connecting each position shows cell movement trajectories within 12 hours.

The Golgi apparatus reorients upon release from elongated confinement. Cells released from rectangular confinement typically migrate along their elongated axis with Golgi on one side of the nucleus instead of in front towards the leading edge. When released from smaller areas $\left(900 \mu \mathrm{m}^{2}\right.$ and $\left.1600 \mu \mathrm{m}^{2}\right)$, Golgi apparatus reorient to the front of the nuclei towards the short edge where active membrane protrusions are leading the cell forwards (Figure 5a, b). However for cells released from larger rectangular islands $\left(2500 \mu \mathrm{m}^{2}\right)$, which exhibit negligible movement out of the pattern, 20 of 27 cells analyzed repositioned their Golgi around the nuclei without establishing a defined polarization (Figure 5c) while the remainder exhibited no repositioning of Golgi (Figure 5d). Integration of the corresponding beta distribution yields an unbiased probability (defined here to be one where cells released from the $2500 \mu \mathrm{m}^{2}$ islands reposition their Golgi without a defined
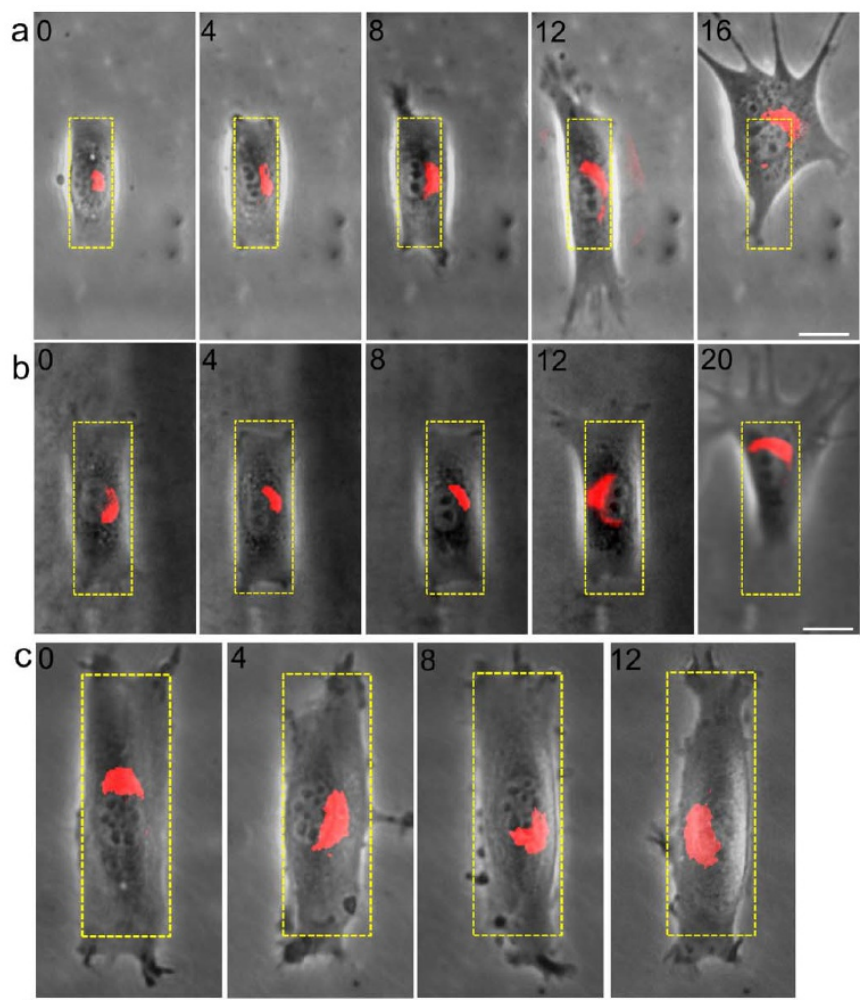

d 0
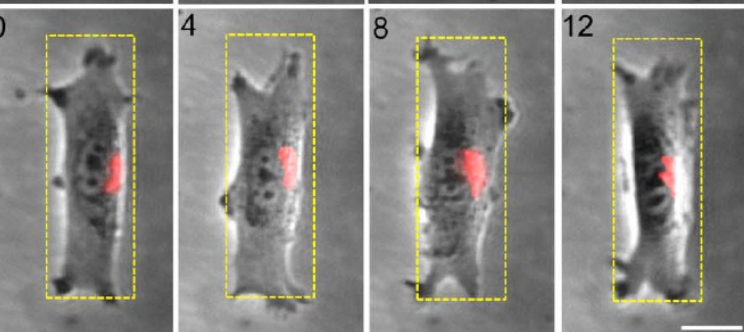

Figure $5 \mid$ Golgi apparatus reorients when the cell is released from confinement. Movement and orientation of the Golgi apparatus of single NIH 3T3 cell released from an adhesive island with an area of (a) $900 \mu \mathrm{m}^{2}$, (b) $1600 \mu \mathrm{m}^{2}$, (c) and (d) $2500 \mu \mathrm{m}^{2}$. The scale bar corresponds to $20 \mu \mathrm{m}$.

polarization between $45 \%$ and $55 \%$ of the time) of $2.3 \%$. These observations show that the Golgi polarity observed in cells does not necessarily determine the directional cell movement, but rather is the result of active cell movement.

\section{Discussion}

Cell shape and spreading have been known to influence the mechanical force balance within mammalian cells ${ }^{20,21}$. Here, we released cells of defined size and shape and demonstrated that cell spreading area alone influences significantly the rate of cell movement upon release. There is a difference between the substrate that the cells are initially confined, i.e., cell culture dish, and the substrate that cells subsequently migrate onto, chitosan, which tempers this finding. The commonly used wound healing assay to measure cell migration rate is a useful tool for studying collective cell movements wherein the average spreading area of cells can also be controlled via the seeding density. However, cell-cell contacts influence the polarity of individual cells $\mathrm{s}^{50}$ and the method is unsuitable for investigating the polarity and movement of individual cells. The micropatterned polyelectrolyte self-assembly method used here to temporally and spatially control cell dimensions provides a tool for understanding the coordinated regulation of cell geometry and movement of individual cells. 
Here, confined cells were released from defined starting geometries to visualize the time-dependent Golgi reorganization and directional protrusions during confinement and after release. Our results show that cells with limited spreading area polarize and migrate out of confinement more rapidly than cells with larger initial spreading area. Fibroblasts released from elongated confinement, which mimic their morphology in $v i v o^{40}$, show that the position of Golgi is dependent on spreading area and morphology of the cell. However, the position of Golgi relative to the nucleus prior to the release does not correspond to the direction of movement upon release. Instead, lamellipodia, which extend more actively from the short edges, consistently define the majority of cell movements along the long axis of the cells.

Only after cells migrate out from confinement does the Golgi apparatus reorient toward the direction of cell movement. This is consistent with previous findings that Golgi apparatus locate toward the leading edge of migrating fibroblasts and neurons $\mathrm{s}^{26,29,36}$. The final front position of the Golgi apparatus provides membranes and proteins from Golgi-derived vesicles necessary to sustain forward movement $^{31,36,37}$.

Earlier experiments with cells confined and released from asymmetric teardrop shapes have suggested that asymmetric geometries can lead to cell polarization as indicated by the localization of the Golgi in front of the blunt edge of the teardrops from where migration initiates ${ }^{48}$. With the current rectangular-shaped patterns, we observed reorientation of Golgi towards the direction of migration. These data suggest that actin organization resulting from the teardrop morphology, rather than Golgi polarization, can lead to the observed asymmetric lamellipodia extension and directional migration.

The results of these controlled experiments on Golgi reorientation dynamics following initial migration imply that Golgi polarization in front of the nucleus is not a prerequisite for directional migration. This idea is supported by a few studies on free migrating cells ${ }^{51}$, and cells migrating along line patterns ${ }^{52}$ that exhibit uncorrelated localization of Golgi and/or MTOC with the migration direction. Cell geometry sets the actin filament organization and membrane protrusions that determine the direction of initial cell movement independent of the position of Golgi. The initial Golgi position does not define the leading edge. Instead, reorientation follows directional movement and the final frontal position suggests that it may be important for maintaining but not initiating directional migration. The time dependent polarization of Golgi apparatus highlights the complex coordination of intracellular structures in response to changes in their extracellular environments. These results motivate more detailed investigation of the spatial and temporal interactions of the intracellular structures and signals in mediating polarity, a process important during morphogenesis and wound healing.

\section{Methods}

Materials. Tissue culture dishes were purchased from Fisher Scientific (catalog no. 430166) and used as received. Polydimethylsiloxane (PDMS; Sylgard 184) was obtained from Dow Corning (Midland, MI) and used at 10:1 (w:w) base: curing agent. Media-soluble chitosan ( $93 \mathrm{kDa}, 74 \%$ deacetylation) was a gift from TriCorporation (Alpharetta, GA). Alexa 488 or 594-phalloidin, 4',6-diamidino-2phenylindole (DAPI), golgin-97 (human), mouse IgG1, monoclonal CDF4 (antiGolgi), CellLight Golgi-RFP *BacMam 2.0, and Alexa Fluor ${ }^{\circledR} 488$ goat anti-mouse IgG1 $(\gamma 1)$ (secondary antibody) were purchased from Molecular Probes (Eugene, OR). Phosphate buffered saline (PBS), Iscove's modified Dulbecco's medium (IMDM), and Fetal bovine serum (FBS) were purchased from Invitrogen (Carlsbad, CA). Platelet-Derived Growth Factor-BB human (PDGF) was obtained from SigmaAldrich (St. Louis, MO).

Preparation of poly (OEGMA-co-MA). Random copolymers of oligo(ethylene glycol) methacrylate (OEGMA) and methacrylic acid (MA) (Scientific Polymer Products, NY) were prepared by free radical polymerizations of $10 \mathrm{wt} \%$ methanolic solutions of the two monomers (80:20 OEGMA to MA mass ratio) at $60^{\circ} \mathrm{C}$ over $24 \mathrm{hrs}$, initated with $1 \mathrm{wt} \%$ (with respect to monomer) 2,2' -azobis(2amidinopropane) dihydrochloride (Wako, VA).
Cell confinement and release. Micropatterns of varying size (900, 1600, and $2500 \mu \mathrm{m}^{2}$ ) and shapes (square, triangle, pentagon, hexagon, and trapezoid) were fabricated on silicon wafers using standard photolithographic techniques. From this silicon master, complementary PDMS replicas were prepared by soft lithography ${ }^{53}$ and used as stamps in subsequent microcontact printing steps to form patterns of poly(OEGMA-co-MA) copolymer directly on cell culture dishes. Patterned dishes were sterilized under UV for one hour prior to plating of cells. Masking of poly(OEGMA-co-MA) printed regions by adsorption of chitosan to render these regions cell-adhesive was accomplished by immersing the culture dish with media containing $0.2 \%$ water-soluble chitosan for 20 minutes $^{39}$. All experiments were designed only to change the geometric confinement of the cells. All other conditions including the nutrient or chitosan properties were maintained constant.

Cell culture. NIH 3T3 fibroblasts were purchased from ATCC and cultured in Iscove's modified Dulbecco's medium (IMDM) supplemented with 10\% FBS. Cultures were maintained at $37^{\circ} \mathrm{C}$ in a humidified atmosphere containing $5 \% \mathrm{CO}_{2}$. Sub-confluent monolayers were dissociated with $0.25 \%$ trypsin-EDTA solution, resuspended in IMDM with 10\% FBS, and then plated on micropatterned culture dishes.

Immunofluorescence staining. Cells were fixed with 3.7\% paraformaldehyde for 15 minutes, washed in phosphate buffered saline, permeabilized with $0.1 \%$ Triton X-100 for 5 minutes and then blocked with 3\% BSA (bovine serum albumin) for 30 minutes. Samples were subsequently rinsed with PBS then incubated with Alexa 594-phalloidin, Alexa 488- conjugated anti-Golgin-97 (human), and DAPI to stain for F-actin, Golgi apparatus, and nuclei respectively. Fixed cells were imaged using Zeiss LSM 710 NLO confocal microscope, $63 \times$ plan apochromatic water objective lens and digitally acquired using Zeiss Meta software version 3.5.

\section{Cell migration and statistical analysis. Cells were plated at a density of}

$\sim 10,000$ cells $/ \mathrm{cm}^{2}$ and allowed to spread within the cell-adhesive patterns of different sizes simultaneously for 12 hours. The cells were then released from confinement by incubating with complete media containing $0.2 \%$ chitosan for 20 minutes, after which the cells were restored in complete media containing no chitosan and with $5 \mathrm{ng} / \mathrm{ml}$ PDGF. Migration following release was tracked with Nikon TE-2000 inverted microscope equipped with CCD camera (SPOT CAM, Diagnostic Instruments Inc.), $20 \times$, NA 0.25 A-plan objective lens. Time-lapse phase contrast images were processed with Metamorph software (Ver 6.0r4, Universal Imaging, Westchester, PA). Only individual cells confined within each individual island were considered for analysis. The cell migration out of the pattern was tracked for 12 hours beyond which cells divisions were observed. Quantification of cell shape and determination of the location of the Golgi were performed using NIH ImageJ and Metamorph software. Statistical analysis on the localization of Golgi toward either the short or long edges were performed assuming a binominal distribution. Significance analysis of angular distributions of Golgi apparatus for fibroblast cells on rectangular patterns with different areas were analyzed taking into account differences in sample variances, i.e., heteroscedastic t-test.

1. Brundage, R. A., Fogarty, K. E., Tuft, R. A. \& Fay, F. S. Calcium gradients underlying polarization and chemotaxis of eosinophils. Science 254, 703-706 (1991).

2. Wei, C. et al. Calcium flickers steer cell migration. Nature 457, 901-905 (2009).

3. Servant, G. et al. Polarization of chemoattractant receptor signaling during neutrophil chemotaxis. Science 287, 1037-1040 (2000)

4. Jin, T., Zhang, N., Long, Y., Parent, C. A. \& Devreotes, P. N. Localization of the G protein beta gamma complex in living cells during chemotaxis. Science 287, 1034-1036 (2000).

5. Schmidt, C. E., Horwitz, A. F., Lauffenburger, D. A. \& Sheetz, M. P. Integrincytoskeletal interactions in migrating fibroblasts are dynamic, asymmetric, and regulated. J. Cell Biol. 123, 977-991 (1993).

6. Lawson, M. A. \& Maxfield, F. R. $\mathrm{Ca}^{2+}$-and calcineurin-dependent recycling of an integrin to the front of migrating neutrophils. Nature 377, 75-79 (1995).

7. Nabi, I. R. The polarization of the motile cell. J. Cell Sci. 112, 1803-1811 (1999).

8. Lauffenburger, D. A. \& Horwitz, A. F. Cell migration: A physically integrated molecular process. Cell 84, 359-369 (1996).

9. Theriot, J. A. \& Mitchison, T. J. Actin microfilament dynamics in locomoting cells. Nature 352, 126-131 (1991)

10. Stossel, T. P. On the crawling of animal cells. Science 260, 1086-1094 (1993).

11. Huttenlocher, A., Sandborg, R. R. \& Horwitz, A. F. Cell adhesion in cell-migration. Curr. Opin. Struct. Biol. 7, 697-706 (1995).

12. Dimilla, P. A., Barbee, K. \& Lauffenburger, D. A. Mathematical model for the effects of adhesion and mechanics on cell migration speed. Biophys. J. 60, 15-37 (1991).

13. Mandeville, J. T. H., Lawson, M. A. \& Maxfield, F. R. Dynamic imaging of neutrophil migration in three dimensions: Mechanical interactions between cells and matrix. J. Leukocyte Biol. 61, 188-200 (1997).

14. Palecek, S. P., Loftus, J. C., Ginsberg, M. H., Lauffenburger, D. A. \& Horwitz, A. F. Integrin-ligand binding properties govern cell migration speed through cellsubstratum adhesiveness. Nature 385, 537-540 (1997). 
15. Giancotti, F. G. \& Ruoslahti, E. Elevated levels of the alpha 5 beta 1 fibronectin receptor suppress the transformed phenotype of Chinese hamster ovary cells. Cell 60, 849-859 (1990).

16. Maheshwari, G., Brown, G., Lauffenburger, D. A., Wells, A. \& Griffith, L. G. Cell adhesion and motility depend on nanoscale RGD clustering. J. Cell Sci. 113, 1677-1686 (2000)

17. Kuijpers, T. W. et al. Freezing adhesion molecules in a state of high-avidity binding blocks eosinophil migration. J. Exp. Med. 178, 279-284 (1993).

18. Dimilla, P. A., Stone, J. A., Quinn, J. A., Albelda, S. M. \& Lauffenburger, D. A. Maximal migration of human smooth muscle cells on fibronectin and type IV collagen occurs at an intermediate attachment strength. J. Cell Biol. 122, 729-737 (1993)

19. Maheshwari, G., Wells, A., Griffith, L. G. \& Lauffenburger, D. A. Biophysical integration of effects of epidermal growth factor and fibronectin on fibroblast migration. Biophys. J. 76, 2814-2823 (1999).

20. Balaban, N. Q. et al. Force and focal adhesion assembly: a close relationship studied using elastic micropatterned substrates. Nat. Cell Biol. 3, 466-472 (2001).

21. Versaevel, M., Grevesse, T. \& Gabriele, S. Spatial coordination between cell and nuclear shape within micropatterned endothelial cells. Nat. Commun. 3, 671 (2012).

22. Chen, C. S., Mrksich, M., Huang, S., Whitesides, G. M. \& Ingber, D. E. Geometric control of cell life and death. Science 276, 1425-1428 (1997).

23. Chen, C. S., Alonso, J. L., Ostuni, E., Whitesides, G. M. \& Ingber, D. E. Cell shape provides global control of focal adhesion assembly. Biochem. Biophys. Res. Commun. 307, 355-361 (2003)

24. Thomas, C. H., Collier, J. H., Sfeir, C. S. \& Healy, K. E. Engineering gene expression and protein synthesis by modulation of nuclear shape. Proc. Natl. Acad. Sci. USA 99, 1972-1977 (2002).

25. Nobes, C. D. \& Hall, A. Rho GTPases control polarity, protrusion, and adhesion during cell movement. J. Cell Biol. 144, 1235-1244 (1999).

26. Magdalena, J., Millard, T. H. \& Machesky, L. M. Microtubule involvement in NIH 3T3 Golgi and MTOC polarity establishment. J. Cell Sci. 116, 743-756 (2003).

27. Kupfer, A., Louvard, D. \& Singer, S. Polarization of the Golgi apparatus and the microtubule-organizing center in cultured fibroblasts at the edge of an experimental wound. Proc. Natl. Acad. Sci. USA 79, 2603-2607 (1982).

28. Tzima, E., Kiosses, W. B., del Pozo, M. A. \& Schwartz, M. A. Localized Cdc42 activation, detected using a novel assay, mediates microtubule organizing center positioning in endothelial cells in response to fluid shear stress. J. Biol. Chem. 278 31020-31023 (2003).

29. Schaar, B. T. \& McConnell, S. K. Cytoskeletal coordination during neuroinal migration. Proc. Natl. Acad. Sci. USA 102, 13652-13657 (2005).

30. Itoh, R. E. et al. Activation of Rac and Cdc42 video imaged by fluorescent resonance energy transfer-based single-molecule probes in the membrane of living cells. Mol. Cell. Biol. 22, 6582-6591 (2002).

31. Bergmann, J. E., Kupfer, A. \& Singer, S. J. Membrane insertion at the leading edge of motile fibroblasts. Proc. Natl. Acad. Sci. USA 80, 1367-1371 (1983).

32. Ridley, A. J. et al. Cell migration: Integrating signals from front to back. Science 302, 1704-1709 (2003).

33. Euteneuer, U. \& Schliwa, M. Mechanism of centrosome positioning during the wound response in BSC-1 cells. J. Cell Biol. 116, 1157-1166 (1992).

34. Gomes, E. R., Jani, S. \& Gundersen, G. G. Nuclear movement regulated by Cdc42, MRCK, myosin, and actin flow establishes MTOC polarization in migrating cells. Cell 121, 451-463 (2005).

35. Gotlieb, A. I., Subrahmanyan, L. \& Kalnins, V. I. Microtubule-organizing centers and cell migration: effect of inhibition of migration and microtubule disruption in endothelial cells. J. Cell Biol. 96, 1266-1272 (1983).

36. Etienne-Manneville, S. Cdc42 - the centre of polarity. J. Cell Sci. 117, 1291-1300 (2004).

37. Etienne-Manneville, S. \& Hall, A. Rho GTPases in cell biology. Nature 420, 629-635 (2002).

38. Co, C. C., Wang, Y. C. \& Ho, C. C. Biocompatible micropatterning of two different cell types. J. Am. Chem. Soc. 127, 1598-1599 (2005).
39. Kumar, G., Meng, J. J., Ip, W., Co, C. C. \& Ho, C. C. Cell motility assays on tissue culture dishes via non-invasive confinement and release of cells. Langmuir 21, 9267-9273 (2005).

40. Li, F., Li, B., Wang, Q.-M. \& Wang, J. H. C. Cell shape regulates collagen type I expression in human tendon fibroblasts. Cell Motil. Cytoskel. 65, 332-341 (2008).

41. Pelham, R. J. \& Wang, Y. L. Cell locomotion and focal adhesions are regulated by substrate flexibility. Proc. Natl. Acad. Sci. USA 94, 13661-13665 (1997).

42. Brock, A. et al. Geometric determinants of directional cell motility revealed using microcontact printing. Langmuir 19, 1611-1617 (2003).

43. Parker, K. K. et al. Directional control of lamellipodia extension by constraining cell shape and orienting cell tractional forces. FASEB J. 16, 1195-1204 (2002).

44. Naumanen, P., Lappalainen, P. \& Hotulainen, P. Mechanisms of actin stress fibre assembly. J. Microsc-Oxford 231, 446-454 (2008).

45. Bhadriraju, K. \& Hansen, L. K. Extracellular matrix-and cytoskeleton-dependent changes in cell shape and stiffness. Exp. Cell Res. 278, 92-100 (2002).

46. Doornaert, B. et al. Time course of actin cytoskeleton stiffness and matrix adhesion molecules in human bronchial epithelial cell cultures. Exp. Cell Res. 287, 199-208 (2003)

47. ChrzanowskaWodnicka, M. \& Burridge, K. Rho-stimulated contractility drives the formation of stress fibers and focal adhesions. J. Cell Biol. 133, 1403-1415 (1996).

48. Jiang, X. Y., Bruzewicz, D. A., Wong, A. P., Piel, M. \& Whitesides, G. M. Directing cell migration with asymmetric micropatterns. Proc. Natl. Acad. Sci. USA 102, 975-978 (2005).

49. Kumar, G., Co, C. C. \& Ho, C. C. Steering cell migration using microarray amplification of natural directional persistence. Langmuir 27, 3803-3807 (2011).

50. Desai, R. A., Gao, L., Raghavan, S., Liu, W. F. \& Chen, C. S. Cell polarity triggered by cell-cell adhesion via E-cadherin. J. Cell Sci. 122, 905-911 (2009).

51. Danowski, B. A., Khodjakov, A. \& Wadsworth, P. Centrosome behavior in motile HGF-treated PtK2 cells expressing GFP-gamma tubulin. Cell Motil Cytoskel. 50, 59-68 (2001)

52. Pouthas, F. et al. In migrating cells, the Golgi complex and the position of the centrosome depend on geometrical constraints of the substratum. J. Cell Sci. 121, 2406-2414 (2008).

53. Qin, D., Xia, Y. \& Whitesides, G. M. Soft lithography for micro-and nanoscale patterning. Nat. Protoc. 5, 491-502 (2010)

\section{Acknowledgments}

This work was supported by the National Institutes of Health (R01EB010043 and R21EB3979) and the National Science Foundation (CBET0928219). We thank Kyu-Shik Mun for assistance with the data analysis.

\section{Author contributions}

B.C., G.K., C.C., and C.H. conceived of the experiment, B.C. and G.K. carried out the experiments, B.C., C.C., and C.H. did the data analysis and manuscript writing.

\section{Additional information}

Supplementary information accompanies this paper at http://www.nature.com/ scientificreports

Competing financial interests: The authors declare no competing financial interests. How to cite this article: Chen, B., Kumar, G., Co, C.C. \& Ho, C. Geometric Control of Cell Migration. Sci. Rep. 3, 2827; DOI:10.1038/srep02827 (2013).

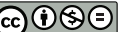

This work is licensed under a Creative Commons AttributionNonCommercial-NoDerivs 3.0 Unported license. To view a copy of this license, visit http://creativecommons.org/licenses/by-nc-nd/3.0 


\title{
SCIENTIFIC REP RTS \\ OPEN Author Correction: Geometric Control of Cell Migration
}

\author{
Bo Chen ${ }^{1}$, Girish Kumar ${ }^{1,2}$, Carlos C. Co ${ }^{1}$ \& Chia-Chi Ho ${ }^{1}$
}

Correction to: Scientific Reports https://doi.org/10.1038/srep02827, published online 3 October 2013

The following information was omitted from the Methods section.

The images in Figures 2, 3B, and 4A were captured through a coverslip. To achieve this, we cut the boundary of tissue culture dishes and mounted with fluoromount-G. The contrast/brightness of the Golgi stain in Figure 5 was adjusted using Image J software which can alter certain details of the Golgi structure. However, the key point was to show the brief position of the Golgi apparatus during the release period. They are not relative to the certain details of the Golgi structure.

(i) Open Access This article is licensed under a Creative Commons Attribution 4.0 International License, which permits use, sharing, adaptation, distribution and reproduction in any medium or format, as long as you give appropriate credit to the original author(s) and the source, provide a link to the Creative Commons license, and indicate if changes were made. The images or other third party material in this article are included in the article's Creative Commons license, unless indicated otherwise in a credit line to the material. If material is not included in the article's Creative Commons license and your intended use is not permitted by statutory regulation or exceeds the permitted use, you will need to obtain permission directly from the copyright holder. To view a copy of this license, visit http://creativecommons.org/licenses/by/4.0/.

(C) The Author(s) 2018

${ }^{1}$ Chemical \& Materials Engineering Program, University of Cincinnati, Cincinnati, OH, 45221-0012, USA. ${ }^{2}$ Division of Biology, Center for Devices and Radiological Health, US Food \& Drug Administration, Silver Spring, MD, 20993, USA. Correspondence and requests for materials should be addressed to C.-C.H. (email: hocc@ucmail.uc.edu) 